\title{
Aberrant lactate dehydrogenase A signaling contributes metabolic signatures in pancreatic cancer
}

\author{
Wenna Jiang $^{1 \#}$, Lu Qiao ${ }^{1 \#}$, Duo Zuo ${ }^{1}$, Di Qin ${ }^{2}$, Jiawei Xiao ${ }^{1}$, Haohua An ${ }^{1}$, Yanhui Wang ${ }^{1}$, Xinwei Zhang $^{1}$, \\ Yu Jin ${ }^{1}, \operatorname{Li}^{\operatorname{Ren}^{1}}$ \\ ${ }^{1}$ Department of Clinical Laboratory, Tianjin Medical University Cancer Institute and Hospital, National Clinical Research Center for Cancer, Key \\ Laboratory of Cancer Prevention and Therapy, Tianjin's Clinical Research Center for Cancer, Tianjin, China; ${ }^{2}$ Tianjin Key Laboratory of Clinical \\ Multi-omics, Airport Economy Zone, Tianjin, China \\ Contributions: (I) Conception and design: W Jiang, L Ren; (II) Administrative support: D Zuo; (III) Provision of study materials or patients: J Xiao, \\ H An; (IV) Collection and assembly of data: L Qiao, D Qin; (V) Data analysis and interpretation: L Qiao, Y Wang, X Zhang, Y Jin; (VI) Manuscript \\ writing: All authors; (VII) Final approval of manuscript: All authors. \\ \#These authors contributed equally to this work. \\ Correspondence to: Li Ren. Department of Clinical Laboratory, Tianjin Medical University Cancer Institute and Hospital, National Clinical Research \\ Center for Cancer, Key Laboratory of Cancer Prevention and Therapy, Tianjin's Clinical Research Center for Cancer, Tianjin 300060, China. \\ Email: liren@tmu.edu.cn.
}

Background: Pancreatic cancer (PC) has the lowest 5-year survival rate; therefore, new early screening methods and therapeutic targets are still urgently required. Emerging technologies such as metabolomicbased liquid biopsy may contribute to the field. We found aberrant lactate dehydrogenase A (LDHA) signaling to be an unfavorable biomarker for PC.

Methods: A total of 9 genes of the glycolysis pathway were detected by enrichment analysis in the PC Gene Expression Omnibus (GEO) dataset. The relationship between LDHA/pyruvate kinase (PKM)/ fructose biphosphate aldolase A (ALDOA)/glyceraldehyde-3-phosphate dehydrogenase (GAPDH) and patient survival was analyzed by Kaplan-Meier plotting analysis of The Cancer Genome Atlas (TCGA). The detection of changing metabolites in the serum of PC patients was performed using a nuclear magnetic resonance (NMR) spectrometer.

Results: We found LDHA was an independent predictor of overall survival (OS) in PC patients $(\mathrm{P}<0.001)$. Consistent with genetic aberrance of LDHA, we identified significant alterations in patients' glycolysisrelated metabolites, including upregulation of lactic acid and downregulation of pyruvic acid. A 0.956 area under the curve (AUC) was achieved using the combinative metabolites score of lactic acid, pyruvic acid, citric acid, and glucose to distinguish PC from healthy controls.

Conclusions: Aberrant LDHA signaling is an unfavorable biomarker for PC and consequential metabolic changes constitute potential diagnostic signatures of PCs.

Keywords: Biomarkers; pancreatic cancer (PC); aerobic glycolysis; gene expression; serum metabolites

Submitted Dec 11, 2020. Accepted for publication Feb 17, 2021.

doi: $10.21037 /$ atm-21-295

View this article at: http://dx.doi.org/10.21037/atm-21-295

\section{Introduction}

Pancreatic cancer (PC) is currently one of the 10 most commonly diagnosed cancer types in the United States (US), and the death rate of $\mathrm{PC}$ is $8 \%$, making it the fourth most fatal cancer after lung, prostate, and colon cancer in men
$(1,2)$. The 5 -year survival rate of patients with this type of cancer is less than $5 \%$ due to presentation at late stages and the absence of early detection and treatment (3). Hence, it is important to explore possible early screening methods and therapies for PC. Many studies have focused on this 


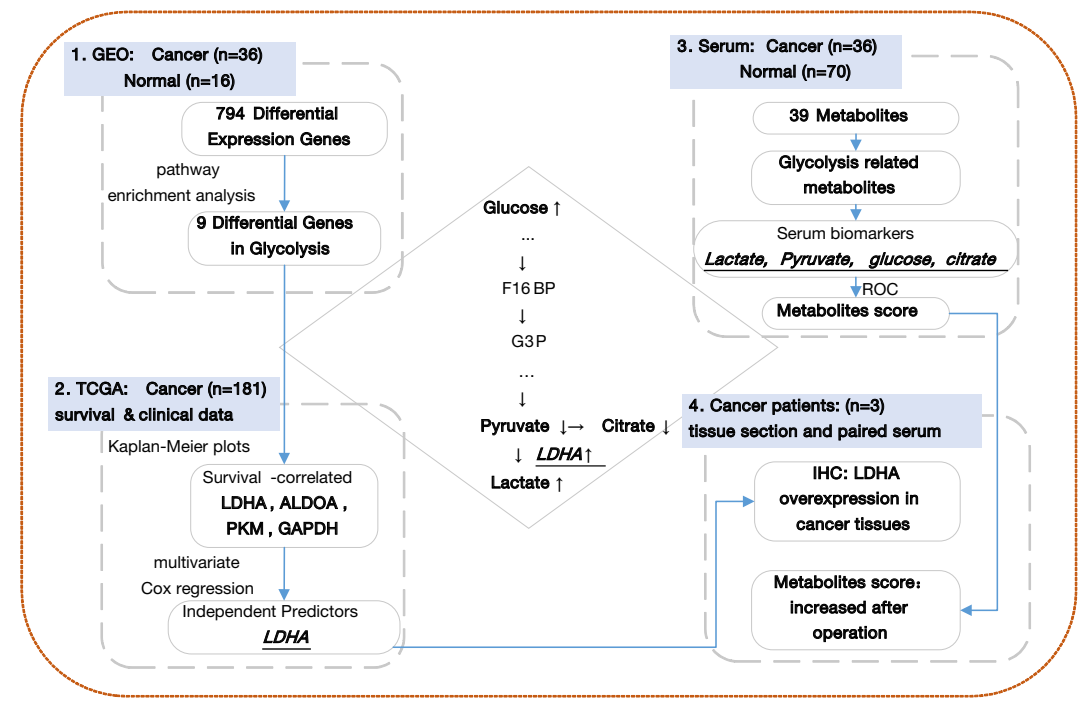

Figure 1 Overall analysis workflow in this article.

topic in recent years, and liquid biopsy is a promising field with great value for application.

Liquid biopsy has the advantages of low invasive sampling, operability, fast turnaround time, and suitability for long-term monitoring. Among biomarkers used in liquid biopsy, metabolomics has the advantages of early diagnosis and treatment development. Metabolites play an important role in human cells and organs and are the key components in maintaining physiological function and intercellular signal transduction, which can reflect the state of the body directly and accurately, while genomics and proteomics are more about what may happen in organisms (4). Therefore, the usage of metabolism is viewed as one of the nextgeneration methodologies of liquid biopsy after genomics $(5-7)$. Tumors have a special metabolism that leads to metabolite alterations in the tumor microenvironment or even bodily status.

Aerobic glycolysis is a metabolic phenomenon that always happens in cancer due to the increased need for energy consumption. Although aerobic glycolysis creates fewer adenosine triphosphates (ATPs) than normal glucose metabolism, cancer cells prefer to use the glycolysis pathway, which is used for energy supply only under anoxic conditions in normal tissue. This progression is known as the "Warburg effect", and leads to the accumulation of lactic acid $(8,9)$.

Although glycolysis has been explored in many types of cancers, metabolic analysis in serum combined with genetic evidence in PC has not been reported. Here, we present the effect of glycolysis in PC on both sides. We analyzed PC and normal tissue gene expression data from Gene Expression Omnibus (GEO) data repositories and verified the differential expression of glycolysis-related genes. Then, The Cancer Genome Atlas (TCGA) dataset was used to analyze the correlation of these genes with the survival time and hazard. Then, serum metabolites were detected to determine the alterations between cancer patients and healthy controls. Glycolysis-related gene expression status and serum metabolite variety in PC patients were combined to explain the relationship between these 2 aspects. The overall workflow of this article is shown in Figure 1. Our results revealed some biomarkers that can promote earlier diagnosis and monitoring of curative effects. We present the following article in accordance with the MDAR checklist (available at http://dx.doi.org/10.21037/atm-21-295).

\section{Methods}

\section{Sample collection}

Venous fasting blood samples of the healthy cohort $(\mathrm{n}=70)$ and PC cohort ( $\mathrm{n}=36)$ were collected at the Tianjin Medical University Cancer Institute and Hospital. All healthy controls included in this study were older than 18 years and healthy upon medical examination. Cancer samples were taken from hospitalized patients before operation, and PC status and clinical stages were confirmed by postoperative 
pathology results.

Blood samples were drawn into serum vacuum tubes and kept at room temperature for 30 minutes before centrifugation $(1,200 \times \mathrm{g}, 10$ minutes), and serum samples were collected into tubes, stored at $80^{\circ} \mathrm{C}$, and shipped in dry ice. The usage of serum samples in this study was reviewed and approved by the Ethics Committee of Tianjin Medical University Cancer Institute and Hospital (Scientific Ethical Approval No. bc2020101) and informed consent was taken from all the patients. The study was conducted in accordance with the Declaration of Helsinki (as revised in 2013).

\section{Gene expression and clinical data of PC patients}

The microarray-based gene expression data of 36 tumor samples and 16 normal samples were obtained from GEO datasets (GSE16515; n=52). Gene expression and detailed clinical data for 181 PC (PAAD) patients were obtained from TCGA (https://cancergenome.nih.gov/abouttcga/ overview). The overall survival (OS) and other PC-related clinical data for each sample were all available in TCGA.

\section{Statistical analyses}

Differentially expressed genes (DEGs) were defined as more than 2-fold change in cancer patients (both upregulation and downregulation). The $\mathrm{P}$ values between cancer and normal cohorts were less than or equal to 0.05 , and pathway enrichment analysis was performed with these DEGs.

Kaplan-Meier plots were used to estimate the correlation between OS and gene expression levels. The median expression value of each gene was selected as the grouping criterion, and cancer patients were divided into high-expression and lowexpression groups. A significant correlation was judged by $\mathrm{P}$ values. Univariate and multivariate Cox regression analyses were all shown in terms of hazard ratios (HRs), estimated 95\% confidence intervals (CIs), and $\mathrm{P}$ values; $\mathrm{P}$ values in the multivariable Cox proportional hazards model were the key indices to identify independent predictors.

The receiver operating characteristic curve (ROC) analysis for the combination of 4 metabolites was calculated using linear regression. The calculated formula was: score $($ all $)=14.143-1.896 \times\left(\right.$ concentration $\left.^{\text {glucose }}\right)-7.812 \times$ $\left(\right.$ concentration $\left.^{\text {citrate }}\right)-2.818 \times\left(\right.$ concentration $\left.^{\text {lactate }}\right)+68.845 \times$ (concentration ${ }^{\text {pyruate }}$ ).

All statistical analyses and data plotting were performed using R software (http:///www.r-project.org). Two-tailed $P$ values were obtained, and a significant difference was defined with a cutoff value of 0.05 .

\section{Serum metabolite detection}

Serum samples were prepared and detected according to Bruker in vitro diagnostic research standard operating procedures (IVDr SOPs) at Protein T Biotechnology Co., Ltd. (Tianjin, China). Briefly, samples were thawed at room temperature, $400 \mu \mathrm{L}$ of serum samples and $400 \mu \mathrm{L}$ of buffer (phosphate buffer $\mathrm{pH} 7.4$ containing TSP-d4, Bruker) were fully mixed, and $600 \mu \mathrm{L}$ of the mixture was transferred to a 5-mm nuclear magnetic resonance (NMR) tube pending analysis. The detection was performed on a $600 \mathrm{MHz}$ NMR Avance III HD spectrometer equipped with a BBI probehead and SampleJet autosampler, which was regulated at $6{ }^{\circ} \mathrm{C}$ during detection (Bruker Biospin, Rheinstetten, Germany). Before acquisition, automatic tuning and shimming were performed on every sample. The free induction decays (FIDs) were presented as spectra after Fourier transformation, and automatic phase and baseline correction were performed in Topspin software as Bruker IVDr. The concentrations of metabolites were expressed as $\mathrm{mmol} / \mathrm{L}$.

\section{Immunobistochemical analysis}

Antibody against LDHA (HuaAn Biotechnology, Huangzhou, China) was used to perform immunohistochemical (IHC) staining in the PC tissues and paired adjacent normal tissues. Briefly, tissue sections were heated at $60^{\circ} \mathrm{C}$ for 25 minutes, de-paraffinized in xylene, and rehydrated using graded alcohol. Hydrogen peroxide $(0.03 \%)$ containing sodium azide was used to block endogenous peroxidase before primary antibody incubation. The sections were then incubated with streptavidin-HRP (streptavidin conjugated to horseradish peroxidase in PBS containing an anti-microbial agent) and 3,3'-diaminobenzidine (DAB)-substrate-chromagen respectively. Positive findings of the IHC staining were observed as a brown coloration in the tissue sections under a light microscope.

\section{Results}

\section{Glycolysis-related differential gene expression in PC}

We analyzed the GEO dataset of PC (GSE16515: 36 cancer samples, 16 normal samples) (10-12) and identified 794 DEGs (fold change (cancer/normal) $>2$ or $<0.5$, $\mathrm{P}<0.05)$. A total of 186 genes were downregulated, while 

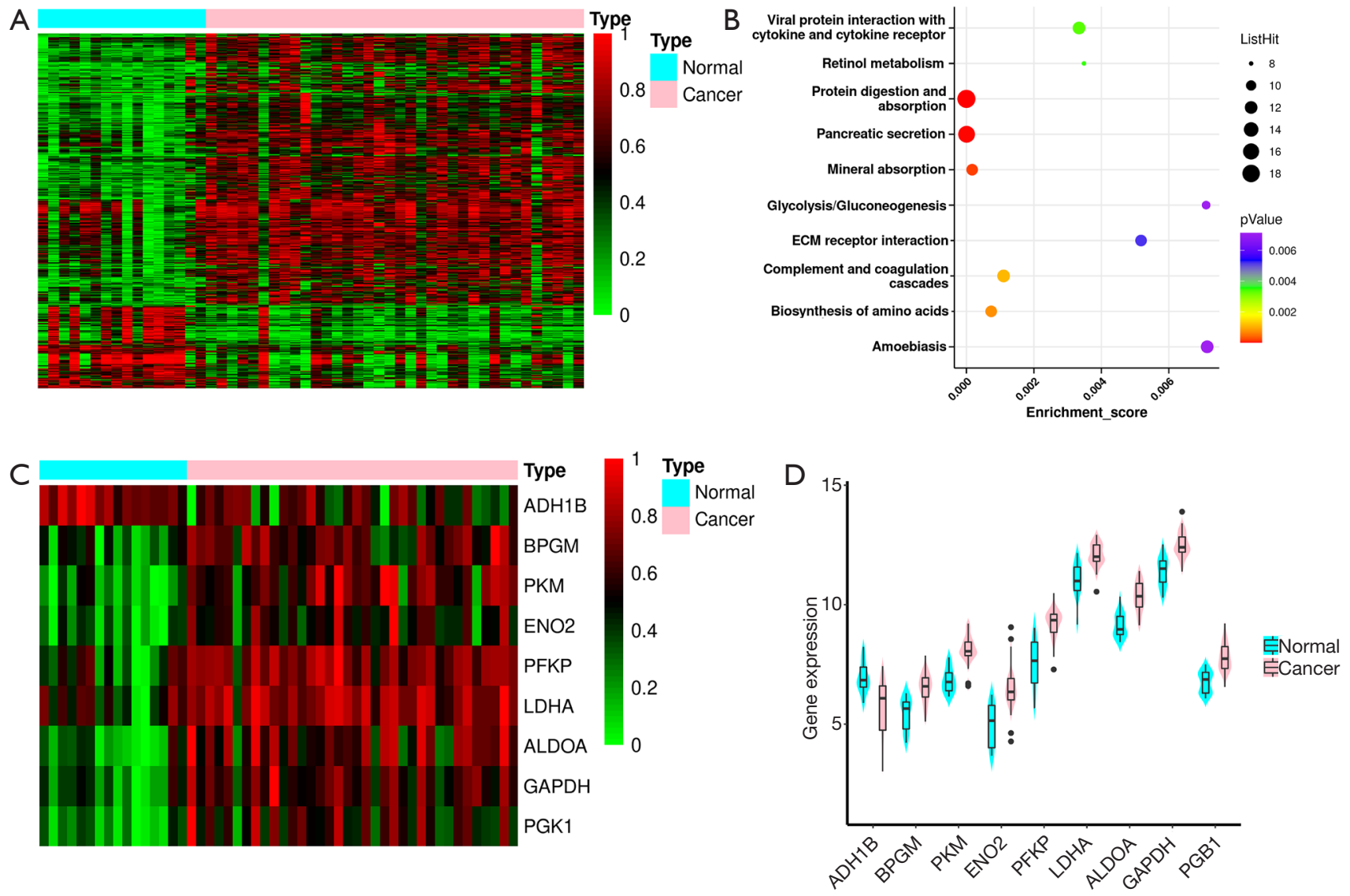

Figure 2 Differential expression and glycolysis-related gene expression in PC. (A) Heatmap analysis of DEGs in the pancreatic cancer GEO dataset. (B) Bubble diagram of the top 10 enriched pathways in KEGG (top 10). (C) Heatmap analysis of glycolysis-related gene expression. (D) The expression levels of 9 genes of the glycolysis pathway in the cancer and normal cohorts in the GEO dataset. PC, pancreatic cancer; DEGs, differentially expressed genes; GEO, Gene Expression Omnibus; KEGG, Kyoto Encyclopedia of Genes and Genomes.

608 genes were upregulated in cancer patients (Figure 2A). Then, we performed pathway enrichment analysis and noticed that 9 genes were enriched in the glycolysis pathway: phosphofructokinase (PFKP), pyruvate kinase (PKM), fructose bisphosphate aldolase A (ALDOA), glyceraldehyde-3-phosphate dehydrogenase (GAPDH), phosphoglycerate kinase (PGK1), lactate dehydrogenase-A (LDHA), bisphosphoglycerate mutase (BPGM), enolase 2 (ENO2), and alcohol dehydrogenase (ADH1B). Except for $\mathrm{ADH} 1 \mathrm{~B}$, the remaining 8 genes were all overexpressed in cancer (Figure 2B,C,D).

\section{Glycolysis-related genes are predictive of prognostic potential}

To determine the relationship between the expression of these 9 genes and survival time in the glycolysis pathway, we analyzed the TCGA PC dataset (181 PC samples). The median expression value of each gene was selected as the grouping criterion, and cancer patients were divided into high-expression and low-expression groups. Kaplan-Meier plots were generated, resulting in a significant correlation between the high expression of 4 genes and lower OS: LDHA $(\mathrm{P}<0.0001)$, ALDOA ( $\mathrm{P}=0.012)$, GAPDH $(\mathrm{P}=0.033)$, and PKM $(\mathrm{P}=0.012)$, as shown in Figure 3. The expression of the other 5 genes was not significantly related to survival probability, as shown in Figure S1.

Moreover, we chose the clinical data in TCGA that are associated with PC risk, as reported (13), such as gender, age, family history of cancer, and history of diabetes, among others. We performed univariate and multivariate Cox regression analyses on these clinical data and 4 survival- 

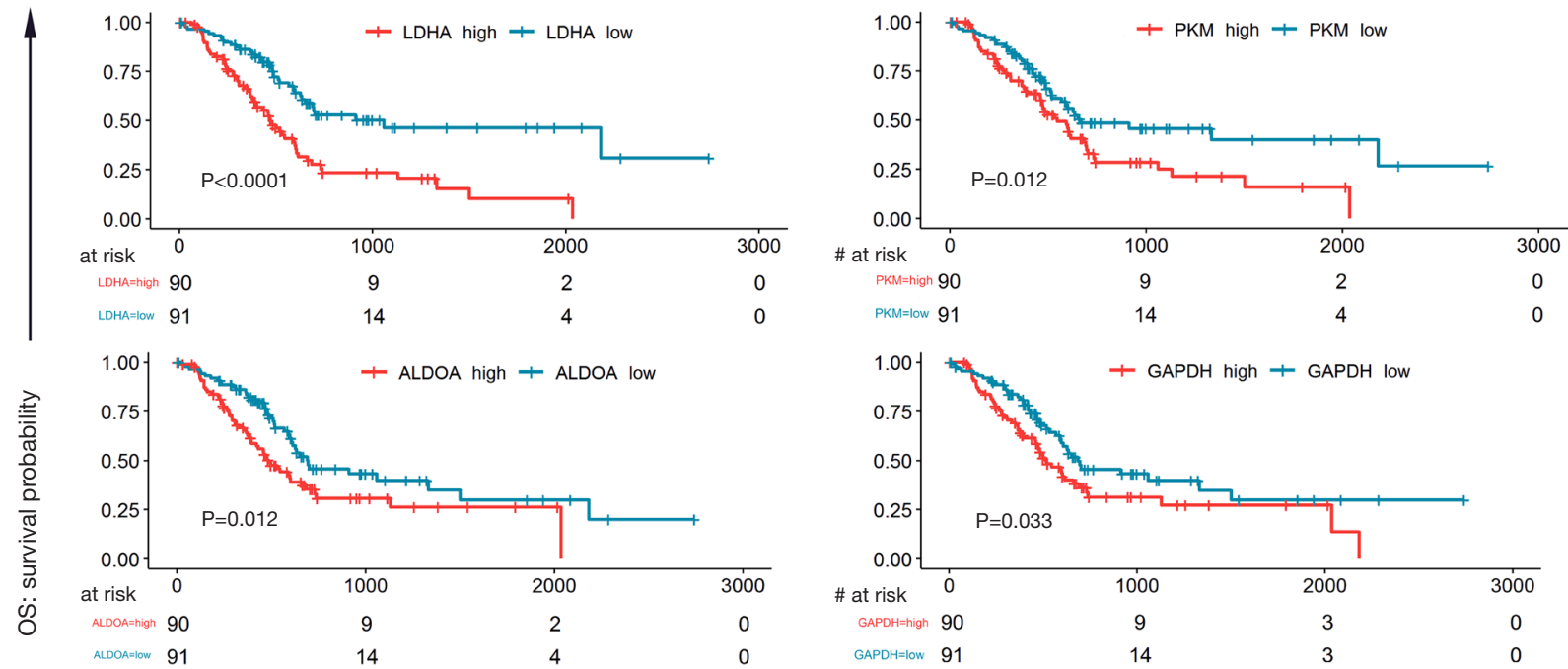

Time (days)

Figure 3 Kaplan-Meier plots of 4 genes in the glycolysis pathway.

related genes in glycolysis (LDHA, ALDOA, GAPDH, PKM). In univariate Cox analysis, age, neoplasm histologic grade, primary therapy outcome success, and the expression of LDHA and PKM were significantly associated with survival time. After multivariate regression analyses, age, primary therapy outcome success, and the expression of LDHA remained remarkable, while neoplasm histologic grade and the expression of PKM were no longer significant (as shown in Table 1). These results identified LDHA expression as an independent predictor of OS in PC patients.

\section{Serum metabolites may reflect glycolysis status in PC}

We tried to determine the metabolite changing characteristics in the serum of PC participants, which would lead to the possibility of early detection or monitoring of cancer recurrence. The detection of metabolites was performed using an NMR spectrometer (Bruker Biospin, Rheinstetten, Germany) in healthy and cancer cohorts. We identified 39 metabolites in serum. The detection values of some metabolites were below the limit of detection (LOD) in more than $80 \%$ of the samples, so we used the remaining 24 metabolites for the following analysis (shown in Table S1). Figure $4 A$ shows the profiling variation of these metabolites, and most were upregulated in cancer. Then, we performed principal component analysis (PCA) on the dataset, and the results showed that the normal and cancer serum samples had distinctive metabolomic profiles (Figure 4B).

Glycolysis-related metabolite concentrations showed upregulation of glucose and lactic acid and downregulation of pyruvic acid and citric acid in cancer patients (Figure 4C). We performed ROC analysis on these 4 metabolites alone and their combinations in cancer and healthy cohorts (Figure 4D). The areas under the curve (AUCs) of these metabolites were 0.670 (citric acid), 0.732 (pyruvic acid), 0.778 (lactic acid), 0.841 (glucose), and was best for the combination score of 0.956 (all). The calculated formula of the combination score was: score $($ all $)=14.143-1.896 \times\left(\right.$ concentration $\left.^{\text {glucose }}\right)-7.812 \times$ $\left(\right.$ concentration $\left.^{\text {citrate }}\right)-2.818 \times\left(\right.$ concentration $\left.^{\text {lactate }}\right)+68.845 \times$ (concentration $^{\text {pyruvate }}$ ). The score was significantly lower (with more negative values) in cancer patients compared with the healthy cohort (shown in Table 2).

\section{LDHA overexpression and metabolites alternation in PC specimens}

We chose another 3 PC patients, and performed IHC staining in the PC and adjacent normal tissues with LDHA antibody. The clinical parameters and contrast-enhanced computed tomography (CT) scans are shown in Figure 5 A. Briefly, pre-operative CT scans showed the tumor location and rough tumor size (the exact size of tumors were measured post-operatively); intraoperatively, the frozen section showed that the mass had features of a tumor of the pancreas with negative resection margins; the CT scans 
Table 1 Univariate and multivariate Cox regression analyses of PC in TCGA

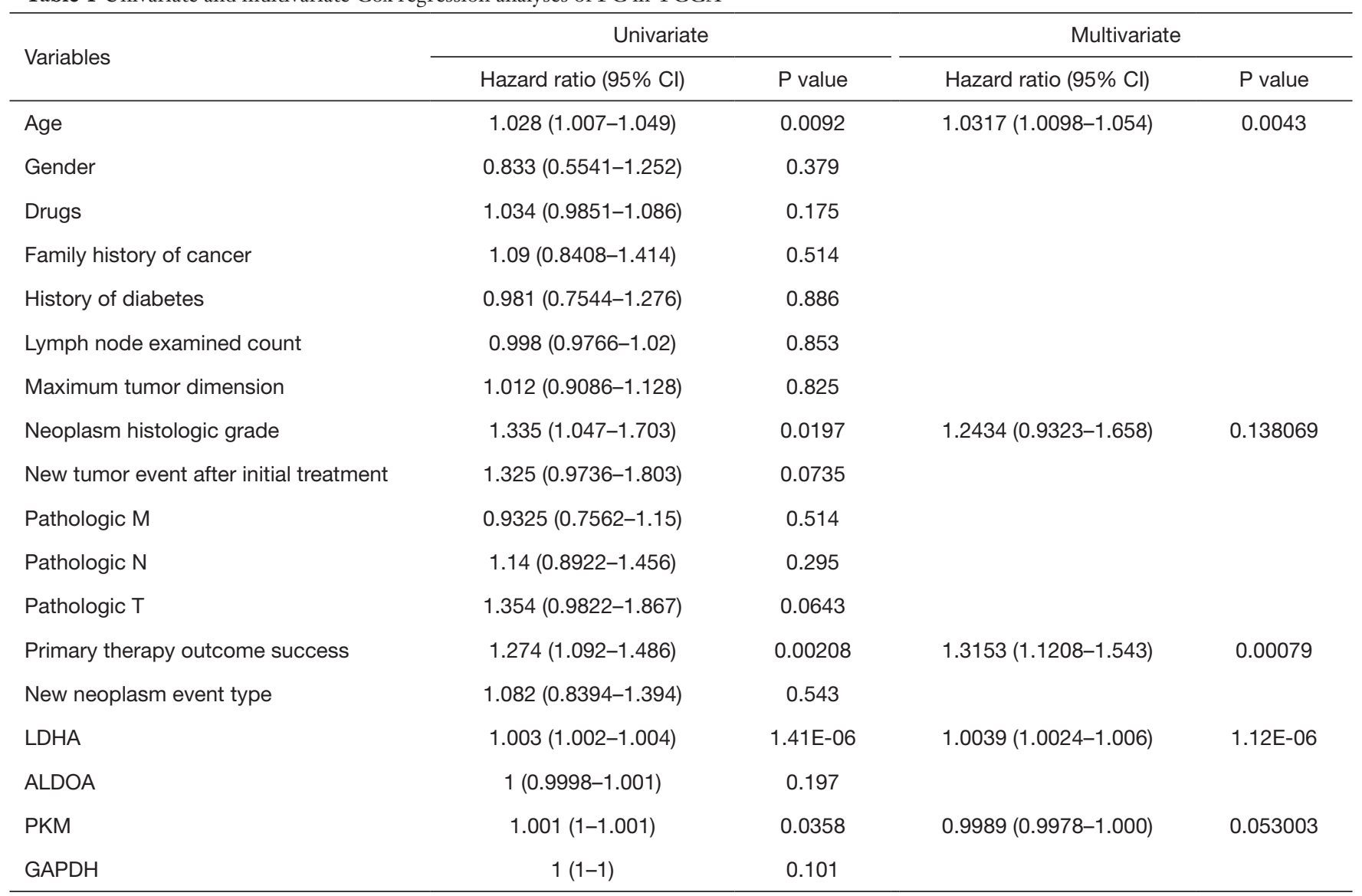

PC, pancreatic cancer; TCGA, The Cancer Genome Atlas; CI, confidence interval; M, metastasis; N, node; T, tumor; LDHA, lactate dehydrogenase-A; ALDOA, fructose bisphosphate aldolase A; PKM, pyruvate kinase; GAPDH, glyceraldehyde-3-phosphate dehydrogenase.

after operation confirmed no cancer was remaining; the PC diagnosis was confirmed by histopathology and IHC after operation. The clinical parameters of these 3 participants are shown in Table 3.

The results of IHC indicated that LDHA was strongly overexpressed in all 3 cancer cases compared with their adjacent normal tissues (Figure 5B). In addition, we collected the serum samples of these 3 participants before and 1 week after their operations, NMR metabolite detection was also performed on these paired samples. The metabolites scores of preoperative samples were all less than -15 , which could be grouped in the cancer cohort, and the scores showed some improvement in postoperative samples (Figure 5C).

\section{Discussion}

Aerobic glycolysis and the Warburg effect have been well studied in recent decades, and their high rates in PC create a hypoxic and nutrient-poor microenvironment $(14,15)$. Glycolysis in PC regulates vigorous tumor growth, invasion, and migration via glycolytic enzymes $(16,17)$. The overexpression of glycolysis enzymes has been reported in many cancer types $(18,19)$. The gene PFKFB4 was suggested to be a promoter of cancer metastasis in breast (20) and bladder cancer (21). Glycolysis induced by TP53 and apoptosis regulator (TIGAR) is associated with cancer progression and poor prognosis in lung cancer $(22,23)$. The overexpression of ALDOA was also associated with poorer prognosis and possibility of metastasis (24), and the silencing of ALDOA in PAN-1 cells decreased proliferation and metastasis, which indicated that ALDOA may become a drug target $(25,26)$. In this article, we found that 9 genes in glycolysis were overexpressed in PC, 4 of which are associated with patient OS. After multivariate 
A

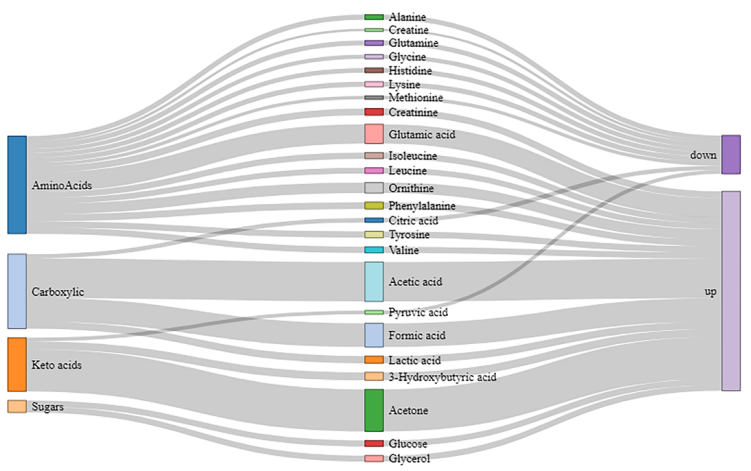

C
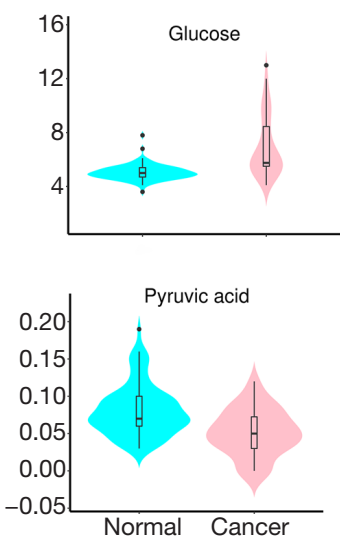

B

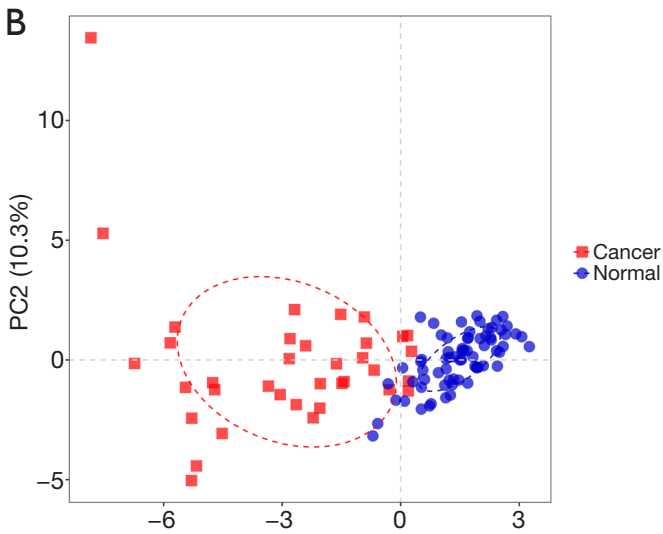

D

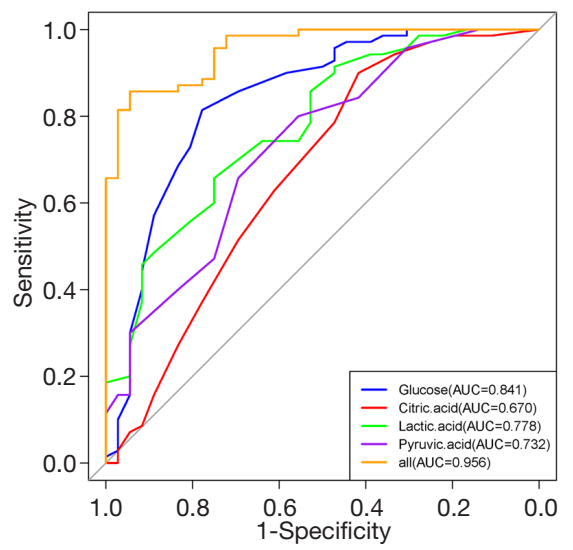

Figure 4 Metabolite changing characteristics in PC serum. (A) Sankey diagram of serum metabolite variety in cancer (cancer/normal). (B) Comparison of the cancer and normal metabolomes via principal component analysis. (C) Violin plot of serum glycolysis-related metabolites in cancer and normal cohorts. (D) ROC curves of glucose, citric acid, lactic acid, pyruvic acid, and their combination (all). PC, pancreatic cancer; ROC, receiver operating characteristic.

Table 2 Glycolysis-related metabolites concentration statistics in $\mathrm{mmol} / \mathrm{L}$

\begin{tabular}{lccc}
\hline Name & $\begin{array}{c}\text { Cancer median } \\
\text { (min-max) }\end{array}$ & $\begin{array}{c}\text { Normal median } \\
\text { (min-max) }\end{array}$ & P value \\
\hline Glucose & $5.75(4.1-13)$ & $5(3.6-7.8)$ & $1.31 \mathrm{E}-05^{\star \star}$ \\
Lactic acid & $2.9(1.6-5.1)$ & $2.1(1-3.9)$ & $5.27 \mathrm{E}-06^{\star \star}$ \\
Pyruvic acid & $0.05(0-0.12)$ & $0.07(0.03-0.19)$ & $2.84 \mathrm{E}-05^{\star \star}$ \\
Citric acid & $0.09(0-0.19)$ & $0.11(0-0.18)$ & $0.0067^{\star}$ \\
Score & $-3.4(-15.3$ to 2.7$)$ & $3.5(-1.7$ to 7.9$)$ & $5.20 \mathrm{E}-11^{\star \star}$ \\
\hline
\end{tabular}

*, $\mathrm{P}<0.05 ;{ }^{*}, \mathrm{P}<0.001$.

Cox regression analyses with PC risk factors, LDHA was identified as an independent predictor of PC.

In glycolysis, LDHA is a very important rate-limiting enzyme. It is the final step in the glycolysis pathway, catalyzing the formation of lactic acid from pyruvic acid, and has been reported to be overexpressed in many cancers; a decrease in LDHA suppresses proliferation and leads to cell death $(27,28)$. There have been more studies on inhibiting LDHA than ALDOA (29,30). Small-molecule inhibitors, such as FX11 [3-dihydroxy-6-methyl-7(phenylmethyl)-4-propylnaphthalene-1-carboxylic acid] and some novel LDHA inhibitors, were used alone or combined with gemcitabine in cell and mouse models, and the results showed that LDHA is a promising therapeutic target for cancer energy metabolism (31-33).

We tested the serum metabolites of cancer patients using NMR spectrometry and found that pyruvic acid and lactic acid were significantly downregulated and upregulated in cancer patients, respectively, which coincided with the 

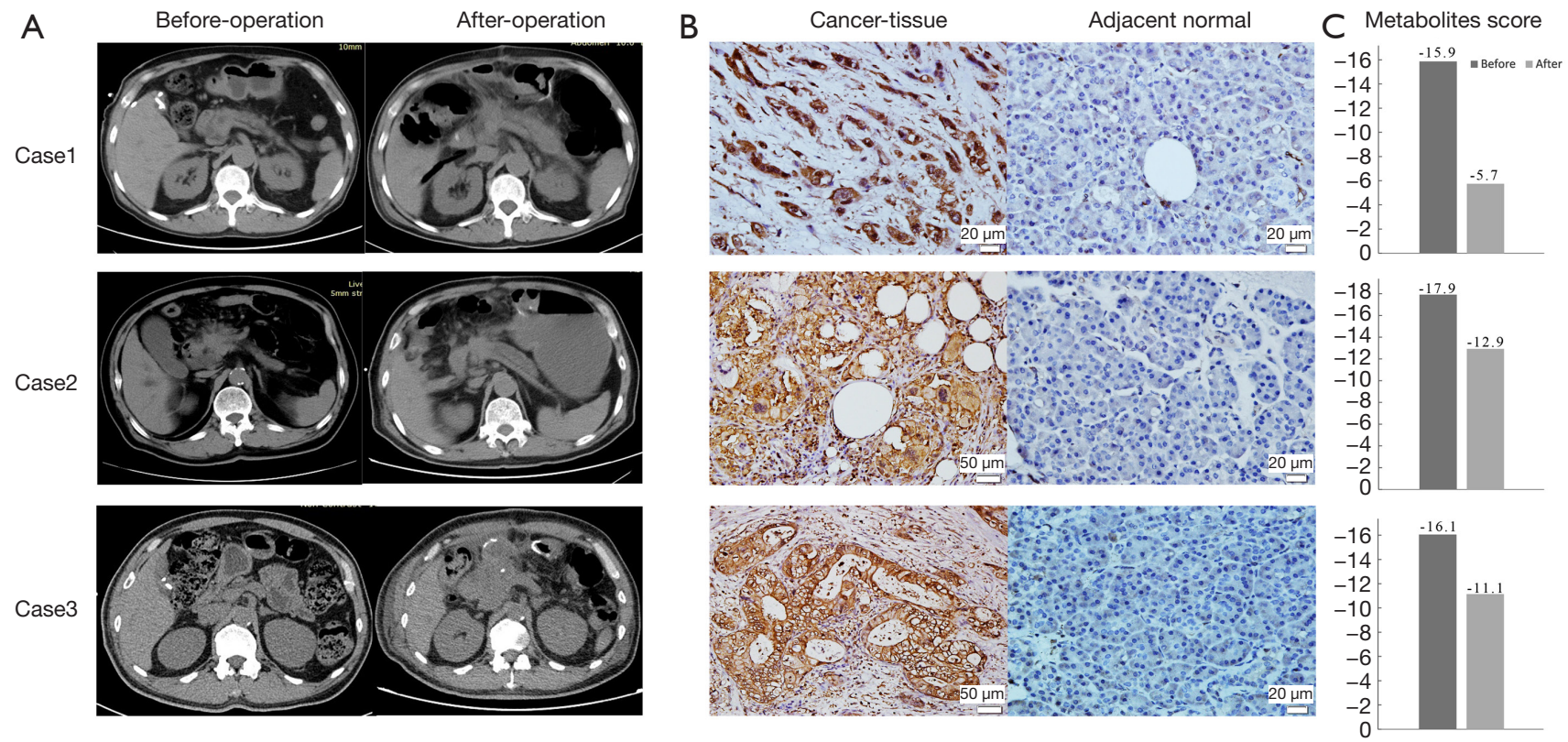

Figure 5 LDHA overexpression and metabolites alternation in pancreatic cancer specimens. (A) Clinical parameters and contrast-enhanced CT scans before and after operation in three cases. (B) IHC staining of LDHA in 3 PC and adjacent normal tissues. Magnification, $\times 200$; scale bars, $50 \mu \mathrm{m}$. (C) Glycolysis-related metabolites score alternation in 3 pancreatic cancer patients before and after operation. LDHA, lactate dehydrogenase-A; CT, computed tomography; IHC, immunohistochemistry; PC, pancreatic cancer.

Table 3 Clinical parameters of 3 cases for IHC

\begin{tabular}{lccc}
\hline Parameters & Case 1 & Case 2 & Case 3 \\
\hline Gender & Male & Male & Male \\
Age & 59 & 67 & 59 \\
Tumor size $(\mathrm{cm})$ & $3 \times 2 \times 1$ & $6 \times 4 \times 3.5$ & Pancreatic head \\
Tumor location & Pancreatic head & Pancreatic body/tail & lb \\
Clinical staging & lb & Ilb & Pancreatic ductal adenocarcinoma \\
Diagnosis & Pancreatic ductal adenocarcinoma & Pancreatic ductal adenocarcinoma (with & osteoclast-like giant cell)
\end{tabular}

IHC, immunohistochemistry.

results of LDHA overexpression (Figure 6). Furthermore, we found that citric acid, the first metabolite in tricarboxylic acid (TCA) that is converted from pyruvic acid, was simultaneously downregulated. At the beginning of glycolysis, the glucose in the serum of our cancer participants was remarkably upregulated. Serum glucose concentration was positively associated with PC (34), and there were reports that high blood glucose levels of PC patients were also associated with poorer outcomes and increased mortality hazard $(35,36)$. The ROC results using these glycolysis-related metabolites indicated that these serum metabolites could be good identifiers of PC; the combinative metabolites score was calculated using lactic acid, pyruvic acid, citric acid, and glucose, and was shown to be significantly higher in healthy cohorts, which may indicate potential as biomarkers for monitoring the curative effects of LDHA inhibitors.

To validate LDHA overexpression and the metabolite results of our study, we collected the surgical tissue sections of another 3 hospitalized patients to perform IHC staining using an LDHA antibody. The IHC results showed that LDHA was significantly overexpressed in 3 cancer tissues 


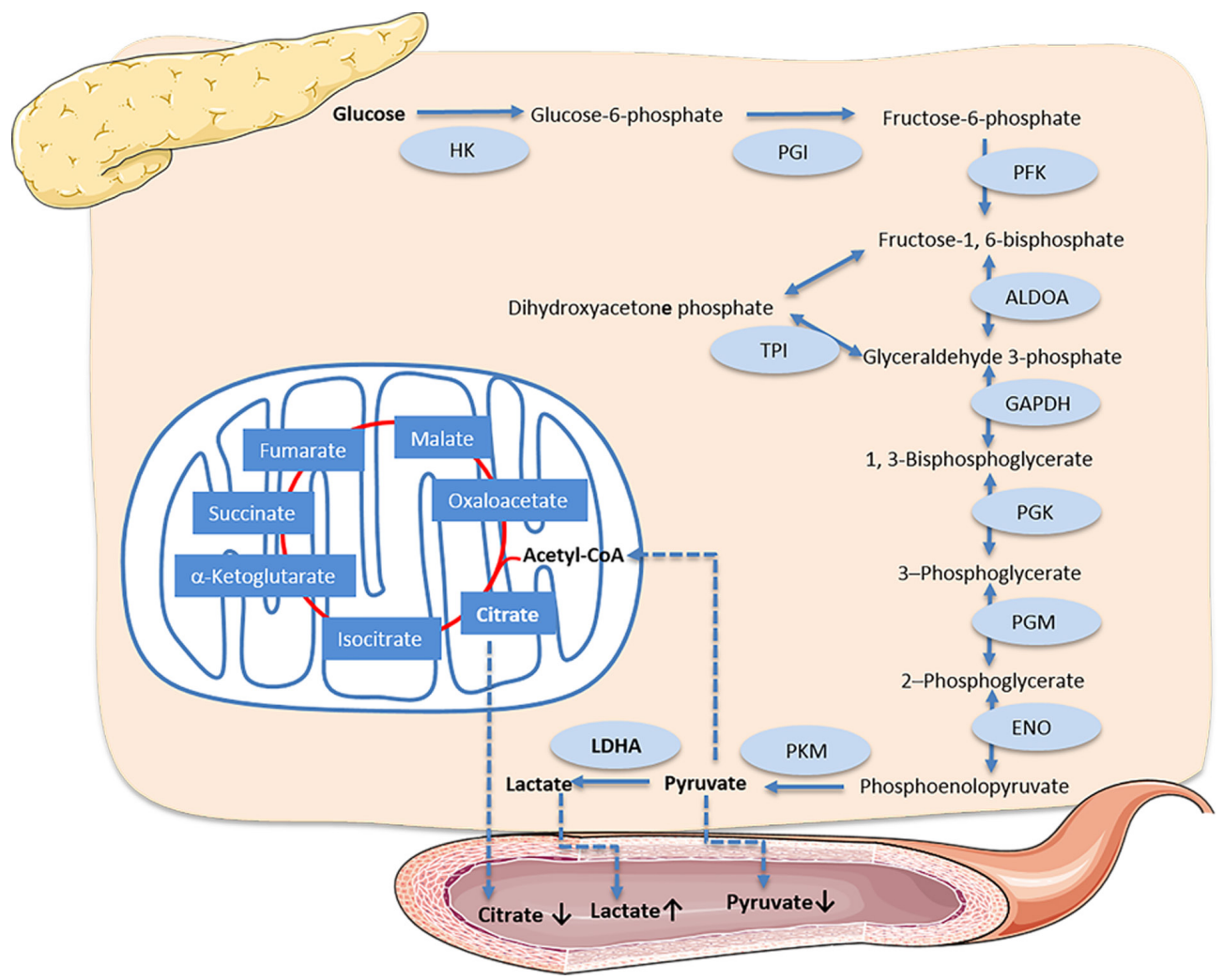

Figure 6 Serum metabolite variety coincides with the overexpression of LDHA. LDHA, lactate dehydrogenase-A.

when compared to their counterparts. We also tested the serum metabolites of these 3 patients before and after surgery. The metabolite scores were calculated, and the scores before operation were all less than -15 , which was less than the minimum score of the healthy cohort in our previous results. These samples could be grouped into the cancer cohort via their glycolysis related metabolite characters; the scores of postoperative samples showed improvement, but had still not recovered to normal status. These results validated the overexpression of LDHA and the glycolysis-related metabolite characteristics revealed in our study.

In this study, we chose the methodology of NMR spectrometer using IVDr system (Bruker Biospin, Rheinstetten, Germany), which has the advantages of excellent reproducibility and accuracy, absence of contamination, high throughput, and lower cost per sample for better preparation and support of clinical screening (37).

\section{Conclusions}

Our results showed that upregulation of lactic acid and downregulation of pyruvic acid coincided with overexpression of LDHA in glycolysis, and the combination of 4 related metabolites (glucose, lactic acid, pyruvic acid, and citric acid) has excellent distinguishing power for PC and can be used as a biomarker for early diagnosis and monitoring of curative effects. However, the sample volume of our research was limited, and the use of these metabolites to monitor curative effects still requires validation through further study.

\section{Acknowledgments}

Funding: This work was supported by the National Natural Science Foundation of China (81402174), the Natural Science Foundation of Tianjin (18JCZDJC32600), the National Natural Science Foundation of China (81802432).

\section{Footnote}

Reporting Checklist: The authors have completed the MDAR checklist. Available at http://dx.doi.org/10.21037/atm-21295 
Data Sharing Statement: Available at http://dx.doi. org/10.21037/atm-21-295

Conflicts of Interest: All authors have completed the ICMJE uniform disclosure form (available at http://dx.doi. org/10.21037/atm-21-295). The authors have no conflicts of interest to declare.

Ethical Statement: The authors are accountable for all aspects of the work in ensuring that questions related to the accuracy or integrity of any part of the work are appropriately investigated and resolved. The usage of serum samples in this study was reviewed and approved by the Ethics Committee of Tianjin Medical University Cancer Institute and Hospital (Scientific Ethical Approval No. bc2020101) and informed consent was taken from all the patients. The study was conducted in accordance with the Declaration of Helsinki (as revised in 2013).

Open Access Statement: This is an Open Access article distributed in accordance with the Creative Commons Attribution-NonCommercial-NoDerivs 4.0 International License (CC BY-NC-ND 4.0), which permits the noncommercial replication and distribution of the article with the strict proviso that no changes or edits are made and the original work is properly cited (including links to both the formal publication through the relevant DOI and the license). See: https://creativecommons.org/licenses/by-nc-nd/4.0/.

\section{References}

1. Siegel RL, Miller KD. Cancer statistics, 2020. CA Cancer J Clin 2020;70:7-30.

2. Gupta R, Amanam I, Chung V. Current and future therapies for advanced pancreatic cancer. J Surg Oncol 2017;116:25-34.

3. Bausch D, Thomas S, Mino-Kenudson M, et al. Plectin-1 as a novel biomarker for pancreatic cancer. Clin Cancer Res 2011;17:302-9.

4. Wishart DS. Emerging applications of metabolomics in drug discovery and precision medicine. Nat Rev Drug Discov 2016;15:473-84.

5. Hanash SM, Ostrin EJ, Fahrmann JF. Blood based biomarkers beyond genomics for lung cancer screening. Transl Lung Cancer Res 2018;7:327-35.

6. Singhal S, Rolfo C, Maksymiuk AW, et al. Liquid Biopsy in Lung Cancer Screening: The Contribution of Metabolomics. Results of A Pilot Study. Cancers (Basel)
2019;11:1069.

7. Yu L, Li K, Zhang X. Next-generation metabolomics in lung cancer diagnosis, treatment and precision medicine: mini review. Oncotarget 2017;8:115774-86.

8. Koppenol WH, Bounds PL, Dang CV. Otto Warburg's contributions to current concepts of cancer metabolism. Nat Rev Cancer 2011;11:325-37.

9. Warburg O, Wind F, Negelein E. THE METABOLISM OF TUMORS IN THE BODY. J Gen Physiol 1927;8:519-30.

10. Pei H, Li L, Fridley BL, et al. FKBP51 affects cancer cell response to chemotherapy by negatively regulating Akt. Cancer Cell 2009;16:259-66.

11. Li L, Zhang JW, Jenkins G, et al. Genetic variations associated with gemcitabine treatment outcome in pancreatic cancer. Pharmacogenet Genomics 2016;26:527-37.

12. Ellsworth KA, Eckloff BW, Li L, et al. Contribution of FKBP5 genetic variation to gemcitabine treatment and survival in pancreatic adenocarcinoma. PLoS One 2013;8:e70216.

13. Vincent A, Herman J, Schulick R, et al. Pancreatic cancer. Lancet 2011;378:607-20.

14. Sancho P, Burgos-Ramos E, Tavera A, et al. MYC/PGC$1 \alpha$ Balance Determines the Metabolic Phenotype and Plasticity of Pancreatic Cancer Stem Cells. Cell Metab 2015;22:590-605.

15. Yang Y, Ishak Gabra MB, Hanse EA, et al. MiR135 suppresses glycolysis and promotes pancreatic cancer cell adaptation to metabolic stress by targeting phosphofructokinase-1. Nat Commun 2019;10:809.

16. Tsutsumi S, Yanagawa T, Shimura T, et al. Autocrine motility factor signaling enhances pancreatic cancer metastasis. Clin Cancer Res 2004;10:7775-84.

17. McDonald OG, Li X, Saunders T, et al. Epigenomic reprogramming during pancreatic cancer progression links anabolic glucose metabolism to distant metastasis. Nat Genet 2017;49:367-76.

18. Qin Y, Cheng C, Lu H, et al. miR-4458 suppresses glycolysis and lactate production by directly targeting hexokinase2 in colon cancer cells. Biochem Biophys Res Commun 2016;469:37-43.

19. Li HM, Yang JG, Liu ZJ, et al. Blockage of glycolysis by targeting PFKFB3 suppresses tumor growth and metastasis in head and neck squamous cell carcinoma. J Exp Clin Cancer Res 2017;36:7.

20. Dasgupta S, Rajapakshe K, Zhu B, et al. Metabolic enzyme PFKFB4 activates transcriptional coactivator SRC-3 to 
drive breast cancer. Nature 2018;556:249-54.

21. Yun SJ, Jo SW, Ha YS, et al. PFKFB4 as a prognostic marker in non-muscle-invasive bladder cancer. Urol Oncol 2012;30:893-9.

22. Shen M, Zhao X, Zhao L, et al. Met is involved in TIGAR-regulated metastasis of non-small-cell lung cancer. Mol Cancer 2018;17:88.

23. Liu J, Lu F, Gong Y, et al. High expression of synthesis of cytochrome c oxidase 2 and TP53-induced glycolysis and apoptosis regulator can predict poor prognosis in human lung adenocarcinoma. Hum Pathol 2018;77:54-62.

24. Cui K, Jin S, Du Y, et al. Long noncoding RNA DIO3OS interacts with miR-122 to promote proliferation and invasion of pancreatic cancer cells through upregulating ALDOA. Cancer Cell Int 2019;19:202.

25. Ji S, Zhang B, Liu J, et al. ALDOA functions as an oncogene in the highly metastatic pancreatic cancer. Cancer Lett 2016;374:127-35.

26. Youns M, ElKhoely A, Kamel R. The growth inhibitory effect of gambogic acid on pancreatic cancer cells. Naunyn Schmiedebergs Arch Pharmacol 2018;391:551-60.

27. Fantin VR, St-Pierre J, Leder P. Attenuation of LDH-A expression uncovers a link between glycolysis, mitochondrial physiology, and tumor maintenance. Cancer Cell 2006;9:425-34.

28. Tachtsidis A, McInnes LM, Jacobsen N, et al. Minimal residual disease in breast cancer: an overview of circulating and disseminated tumour cells. Clin Exp Metastasis 2016;33:521-50.

29. Xintaropoulou C, Ward C, Wise A, et al. A comparative analysis of inhibitors of the glycolysis pathway in breast and ovarian cancer cell line models. Oncotarget

Cite this article as: Jiang W, Qiao L, Zuo D, Qin D, Xiao J, An H, Wang Y, Zhang X, Jin Y, Ren L. Aberrant lactate dehydrogenase A signaling contributes metabolic signatures in pancreatic cancer. Ann Transl Med 2021;9(4):358. doi: 10.21037/atm-21295
2015;6:25677-95.

30. Boudreau A, Purkey HE, Hitz A, et al. Metabolic plasticity underpins innate and acquired resistance to LDHA inhibition. Nat Chem Biol 2016;12:779-86.

31. Rajeshkumar NV, Dutta P, Yabuuchi S, et al. Therapeutic Targeting of the Warburg Effect in Pancreatic Cancer Relies on an Absence of p53 Function. Cancer Res 2015;75:3355-64.

32. Maftouh M, Avan A, Sciarrillo R, et al. Synergistic interaction of novel lactate dehydrogenase inhibitors with gemcitabine against pancreatic cancer cells in hypoxia. Br J Cancer 2014;110:172-82.

33. Le A, Cooper CR, Gouw AM, et al. Inhibition of lactate dehydrogenase A induces oxidative stress and inhibits tumor progression. Proc Natl Acad Sci U S A 2010;107:2037-42.

34. Stolzenberg-Solomon RZ, Graubard BI, Chari S, et al. Insulin, glucose, insulin resistance, and pancreatic cancer in male smokers. JAMA 2005;294:2872-8.

35. Xiao Y, Yang H, Lu J, et al. Serum gammaglutamyltransferase and the overall survival of metastatic pancreatic cancer. BMC Cancer 2019;19:1020.

36. Iarrobino NA, Gill BS, Klement RJ, et al. The Impact of Serum Glucose in the Treatment of Locoregionally Advanced Pancreatic Cancer. Am J Clin Oncol 2019;42:692-7.

37. Jiménez B, Holmes E, Heude C, et al. Quantitative Lipoprotein Subclass and Low Molecular Weight Metabolite Analysis in Human Serum and Plasma by $1 \mathrm{H}$ NMR Spectroscopy in a Multilaboratory Trial. Anal Chem 2018;90:11962-71. 

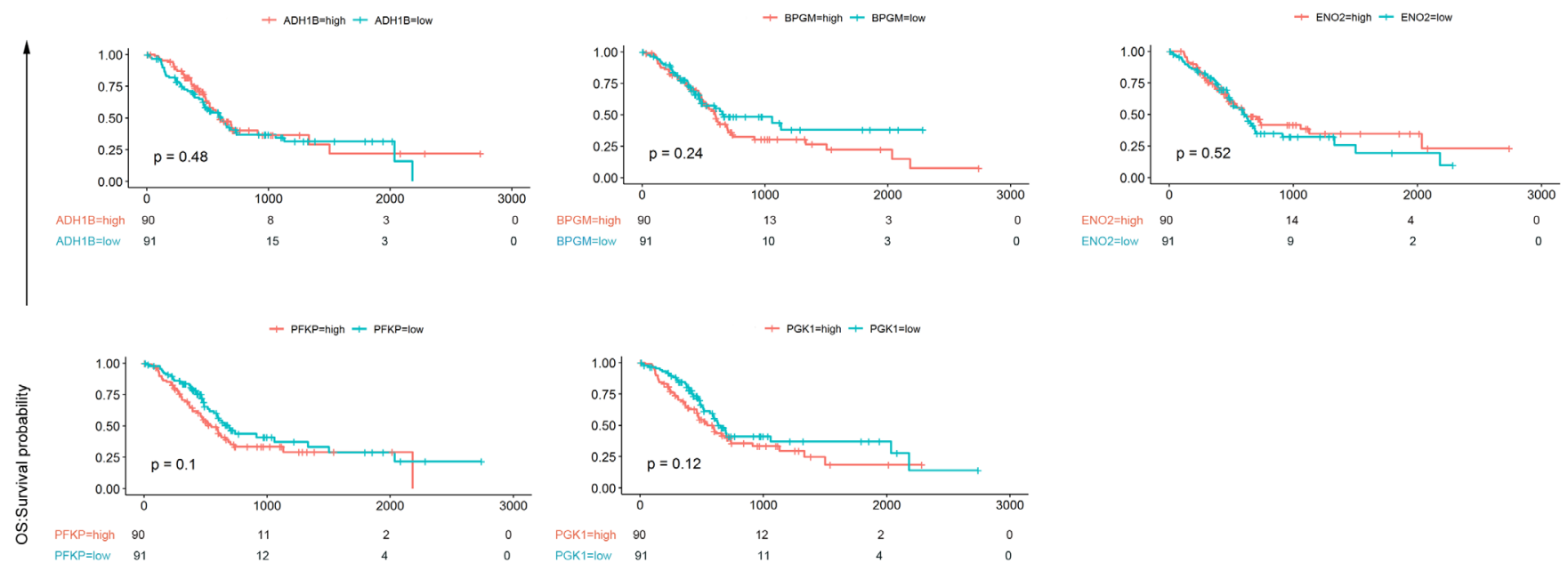

Time (days)

Figure S1 Kaplan-Meier plots of another 5 genes in the glycolysis pathway. 
Table S1 Metabolite concentrations of cancer and healthy cohorts in $\mathrm{mmol} / \mathrm{L}$

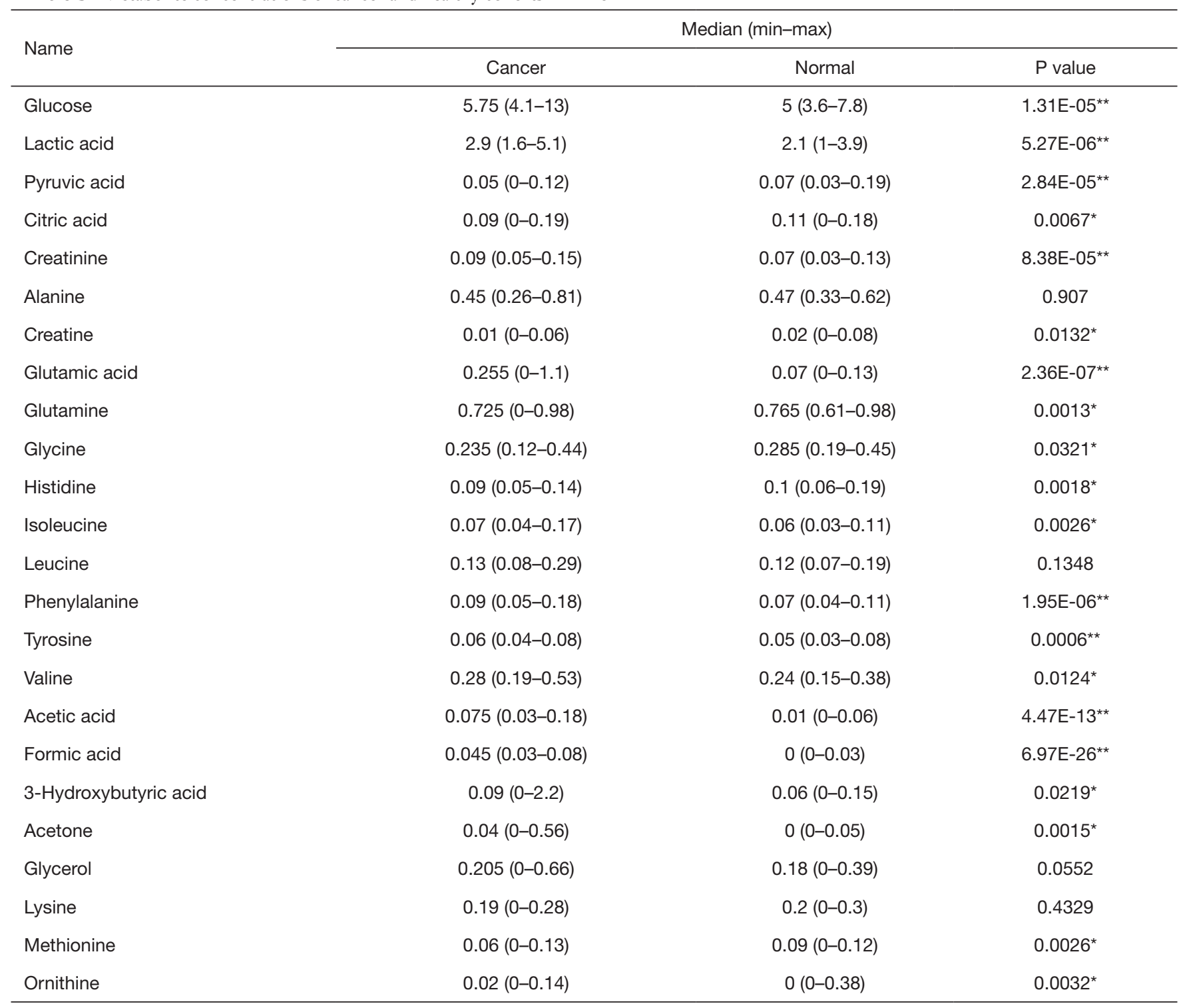

${ }^{*}, \mathrm{P}<0.05 ;{ }^{* *}, \mathrm{P}<0.001$. 